\title{
La teoría de juegos conductual, el dilema del viajero alternativo y la maximización de pagos*
}

\author{
Behavioral game theory, the alternative traveler's dilemma and payoff \\ maximization
}

\author{
RODRIGO MORO** \\ ESTEBAN FREIDIN*** \\ FERNANDO TOHMÉ**** \\ MARCELO AUDAY*****
}

\begin{abstract}
Resumen
Dentro del área de teoría de juegos conductual nos enfocamos en un contexto específico, el del juego que denominamos Dilema del viajero alternativo. En este contexto hemos observado que los participantes tienden a elegir estrategias estrictamente dominadas. Para explicar tendencias similares en otros juegos, los investigadores del área han apelado a sesgos cognitivos o bien a factores motivacionales. Proponemos un factor psicológico denominado sesgo en la accesibilidad de objetivos para explicar las tendencias en cuestión. Ese factor involucra tanto aspectos cognitivos como motivacionales. Presentamos una síntesis de resultados experimentales que favorecen dicha explicación sobre otras alternativas más frugales.
\end{abstract}

Palabras clave: Teoría de juegos, Experimentos, Maximización, Dilema del viajero alternativo, Objetivos.

Clasificación JEL: $C 7, C 91$.

* Este trabajo fue subvencionado por la Agencia Nacional de Promoción Científica y Tecnológica (PICT 106), por el Consejo Nacional de Investigaciones Científicas y Técnicas (PIP 112-201001-00187) y por la Universidad Nacional del Sur (PGI 24/ ZI38). Agradecemos a Federico Contiggiani, Maximiliano Miranda Zanetti y Gustavo Bodanza por ayuda invaluable en todos los aspectos de nuestra investigación. Finalmente, queremos expresar nuestra gratitud hacia un referee anónimo quien nos brindó valiosos comentarios y sugerencias que contribuyeron al mejoramiento de una previa versión del artículo.

** [Autor de correspondencia] Departamento de Humanidades, Universidad Nacional del Sur, Argentina. E-mail: rmoro@uns.edu.ar

*** Centro de Recursos Naturales Renovables de la Zona Semiárida / CONICET, Argentina. E-mail: efreidin@criba.edu.ar

**** Departamento de Economía, Universidad Nacional del Sur / CONICET, Argentina. E-mail: ftohme@criba.edu.ar

****** Departamento de Humanidades, Universidad Nacional del Sur, Argentina. E-mail: ccauday@criba.edu.ar 


\begin{abstract}
Within the area of Behavioral game theory, we focus on a specific context, namely, on a game we called the Alternative traveler's dilemma. In this context, we observe that participants tend to choose strictly dominated strategies. In order to explain similar tendencies in other games, researchers in the area have postulated either cognitive biases or motivational factors. We postulate a psychological factor called "goal accessibility bias" to explain the tendencies at stake. This factor involves both cognitive and motivational aspects. We survey a series of experimental results that favor such an explanation over more frugal alternatives.
\end{abstract}

Key words: Game theory, Experiments, Maximization, Alternative traveler's dilemma, Goals.

JEL Classification: $C 7, C 91$.

\title{
1. INTRODUCCIÓN
}

La literatura del área de Teoría de Juegos Conductual (en inglés, "Behavioral Game Theory") muestra una gran variedad de contextos donde los participantes tienden a elegir estrategias estrictamente dominadas (ej., cooperar en el dilema del prisionero, véanse compendios en Camerer, 2003 y Colman, 2003). Para dar cuenta de estas tendencias, los investigadores del área han apelado a dos tipos distintos de explicaciones: en ciertos contextos se postulan sesgos de tipo cognitivo; en otros contextos, en cambio, se postula la intervención de factores de tipo motivacional. Un ejemplo del primer tipo, que podríamos llamar explicaciones cognitivistas, es el estudio de Camerer et al. (2004) quienes modelan la capacidad limitada de realizar pasos de razonamientos sucesivos y eso les permite dar cuenta de una gran variedad de tendencias conductuales anómalas, por ej., la de no elegir la única estrategia dominante en el juego del concurso de belleza (en inglés, "beauty contest game") ${ }^{1}$. La explicación de Camerer et al. -como las otras del mismo tipo- consiste en postular ciertas creencias y ciertas limitaciones cognitivas. Es importante notar que, sin embargo, es un modelo del tipo de "mejor respuesta", en el sentido de que supone que el agente da su mejor respuesta en busca de la maximización de su propio pago, dadas sus creencias y limitaciones cognitivas. En otras palabras, no se apela a ningún factor motivacional alternativo al modelo estándar de teoría de juegos. Por otra parte, en otros contextos se han postulado explicaciones de tipo motivacional. Un ejemplo claro

$1 \quad$ El juego del concurso de belleza consiste en que todos los participantes elijan simultáneamente un número del 0 al 100. Gana aquel jugador que más se aproxime a los $2 / 3$ del promedio. Este juego tiene un único equilibrio de Nash, a saber, que todos jueguen " 0 ", pero el resultado típico es que sólo una pequeña minoría elige tal estrategia (véase, por ejemplo, Nagel, 1995). 
es el estudio de Fehr y Fischbacher (2002), quienes apelan a preferencias sociales como la reciprocidad para dar cuenta de ciertas tendencias hacia estrategias dominadas en el juego del ultimátum ${ }^{2}$. En efecto, los participantes en este juego suelen rechazar ofertas bajas (de menos del 20\%), pese a que con eso eligen una estrategia dominada y se pierden de maximizar sus propias ganancias. Nótese que aquí se apela a un factor motivacional sin involucrar en absoluto aspectos cognitivos. Se asume (plausiblemente, creemos) que los participantes entienden correctamente la estructura de juego y sus consecuencias.

En este ensayo nos enfocamos en un contexto nuevo, el del dilema del viajero alternativo (DVA) e intentamos mostrar que los tipos de explicaciones mencionadas en el párrafo anterior, tomadas de manera aislada, no pueden dar cuenta de las tendencias hacia estrategias dominadas en este juego. Es así que propondremos una explicación que apela a ambos aspectos, motivacionales y cognitivos considerados conjuntamente, para dar cuenta de las tendencias de elección en cuestión. Más específicamente, postularemos la variable denominada accesibilidad del objetivo de maximización de pagos para explicar las tendencias de elección aparentemente irracionales en el DVA.

Este trabajo está organizado de la siguiente manera. En la sección 2 presentamos el DVA. En la sección 3 resumimos los resultados experimentales de un estudio preliminar y un primer experimento, donde queda al descubierto la participación de aspectos motivacionales en la conducta de los participantes frente al DVA. En la sección 4 resumimos los resultados de un segundo experimento donde quedan al descubierto aspectos cognitivos que afectan la respuesta al juego. En la sección 5 presentamos nuestra propuesta de explicación y discutimos otras alternativas. Finalmente, en la sección 6 presentamos nuestras conclusiones.

\section{El Dilema del Viajero Alternativo3}

El contexto de decisión en cuestión es el DVA y consiste en el siguiente juego:

2 El juego del ultimátum es un juego donde dos jugadores tienen que dividir entre ellos una suma de dinero dada por el experimentador. El jugador 1 debe hacer una propuesta de división y el jugador 2 o acepta la oferta y se asignan los pagos de la manera indicada por el jugador 1 o rechaza la oferta y ambos jugadores se quedan sin pagos. El único equilibrio de Nash está dado por la combinación donde el jugador 1 divide la suma de dinero de manera tal que el jugador 2 recibe sólo la unidad mínima de dinero y el resto va para él mismo y el jugador 2 acepta tal propuesta. Nuevamente, sólo un porcentaje mínimo de participantes juega de la manera predicha (véase, por ejemplo, Henrich et al., 2004).

3 Este juego es una versión alternativa del dilema del viajero original propuesto por Basu (1994). La razón detrás del nombre del juego es que Basu ilustra el juego con la historia de dos viajeros que compran dos reliquias idénticas en una isla lejana y la aerolínea se las rompe en el camino. Así, un representante de la aerolínea diseña el juego en cuestión para decidir cómo compensar a los viajeros. En la versión original, los pagos se hacían en función del menor valor propuesto, mientras que en la versión alternativa (sobre la cual trabajamos), los pagos se hacen en función del valor que cada jugador elige. 
Imagine que usted y otro participante van a jugar a un juego con las siguientes reglas:

- Usted tiene que elegir un solo valor que se puede encontrar entre \$180 y \$ 300 (los extremos pueden elegirse también).

- El otro participante hace lo mismo, pero no se permite ningún tipo de comunicación entre usted y la otra persona, es decir, que usted no sabe qué valor eligió el otro participante.

- En el caso de que el otro participante y usted elijan cantidades distintas se procede de la siguiente manera:

- El participante que dijo el número MENOR se le paga la cantidad que dijo MÁS \$ 5 .

- El participante que dijo el número MAYOR se le paga la cantidad que dijo MENOS \$ 5.

- En el caso de que usted y el otro participante elijan la misma cantidad, se les paga exactamente esa cantidad a ambos.

¿Qué valor elige?

El DVA puede caracterizarse a través de la función de pagos de los jugadores. Siguiendo la convención de llamar a un jugador $i$ y al otro $-i$, la función de pagos del primero, $\pi_{i}$ se puede describir en términos de una función auxiliar $V$, que a cualquier proposición asigna el valor 1 si es verdadera y 0 si es falsa. Las proposiciones a las cuales $V$ valuará se refieren a las declaraciones de ambos jugadores, $s_{i}$ y $s_{-i}$ en el intervalo $[180,300]$. Entonces, en el DVA, el pago de $i$ es:

$$
\pi_{\mathrm{i}}\left(\mathrm{s}_{\mathrm{i}}, \mathrm{s}_{-\mathrm{i}}\right)=\mathrm{s}_{\mathrm{i}}+5\left[\mathrm{~V}\left(\mathrm{~s}_{-\mathrm{i}}>\mathrm{s}_{\mathrm{i}}\right)-\mathrm{V}\left(\mathrm{s}_{\mathrm{i}}>\mathrm{s}_{-\mathrm{i}}\right)\right]
$$

Es fácil de constatar que cualquier $s_{i}$ en $[180,289]$ es una estrategia dominada 4 . De manera menos técnica, nótese que jugar 300 asegura un pago mínimo de 295, mientras que jugar 289 implica un pago máximo de 294. Por lo tanto, 300 domina estrictamente a 289. Este juego no tiene equilibrios de Nash en estrategias puras pero, como mencionamos anteriormente, cualquier estrategia entre 180 y 289 está estrictamente dominada por alguna de las estrategias en el grupo 290-300 (290 está débilmente dominada por 300) ${ }^{5}$. Por lo tanto, lo razonable aquí es no jugar ninguna estrategia por debajo de 290. Para verlo más claramente, uno podría (erróneamente) pensar que (300-300) sería un equilibrio. Sin embargo, no lo es, ya que ambos jugadores tendrían incentivo a bajar una unidad, para obtener el bonus de \$ 5 y quedarse con $\$ 304$. Y nuevamente, si uno cree que el otro jugador va a elegir 299, se tendría el incentivo a jugar 298 y así sucesivamente. Sin embargo, hay un punto de quiebre en 290. Si uno cree

$4 \quad$ En el DV original, en cambio, la función de pago es: $\pi_{i}\left(s_{i}, s_{-i}\right)=\operatorname{Min}\left(s_{i}, s_{-i}\right)+5\left[V\left(s_{-i}>\right.\right.$ $\left.\left.s_{i}\right)-V\left(s_{i}>s_{-i}\right)\right]$, donde $\operatorname{Min}\left(s_{i}, s_{-i}\right)$ elige el menor de los dos valores. El único equilibrio de Nash es, en este caso, el perfil de estrategias $\left(s_{i}, s_{-i}\right)=(180,180)$.

5 Por supuesto, el juego tiene equilibrios en estrategias mixtas que podrían ser calculados fácilmente (siempre combinaciones dentro del rango [290-300]). Sin embargo, estos equilibrios no son fácilmente interpretables empíricamente, por lo cual no se suelen tener en cuenta en la literatura del área. 
que el otro jugador elegirá 290, ya no conviene jugar 289 sino 300, ya que así se obtendrían \$ 295 en lugar de \$ 294.

Ahora bien, en estudios experimentales reportados recientemente (véanse los detalles en Freidin et al., 2009; Moro, Bodanza y Auday, 2010; Auday y Moro, 2010; Moro, Freidin y Tohmé, 2011) mostramos que una gran proporción de personas (entre el 52 y $65 \%$ ) tiende a jugar estrategias dominadas en el DVA, es decir, estrategias en el rango 180-289, perdiéndose de maximizar sus beneficios monetarios. La pregunta clave es, por supuesto, cómo explicar esta tendencia.

\section{Resumen de Estudio Preliminar y Experimento I: Aspecto Motivacional}

Para comenzar a dar cuenta del comportamiento en el DVA, comentaremos los resultados experimentales sobre el fenómeno que parecen arrojar luz sobre las potenciales interpretaciones mencionadas ${ }^{6}$.

En un primer estudio preliminar (realizado con incentivos hipotéticos, i.e., sin incentivos monetarios, véase Freidin et al., 2009 y Moro, Bodanza y Auday, 2010) hicimos que los participantes $(\mathrm{N}=48)$, después de responder al dilema, listaran los pensamientos que habían pasado por su mente mientras tomaban la decisión. Casi todos los participantes mencionaron el o los objetivos que perseguían con su elección. Sin embargo, se halló una diferencia importante: los que jugaban estrategias dominantes tendían a mencionar el objetivo de maximizar pagos, en oposición a los que jugaban estrategias dominadas que tendían a mencionar otro tipo de objetivos, muchos de los cuales eran de naturaleza competitiva (ej., ganar el premio, evitar el castigo y ganarle al otro, en el sentido de ganar el premio y más dinero que el otro). Una hipótesis que surgió a partir de esos datos es que muchos participantes podrían haber elegido estrategias estrictamente dominadas -i.e., por debajo del límite de 290- en el afán de satisfacer algún objetivo competitivo (como los señalados más arriba). Claramente, esta hipótesis del objetivo competitivo podría tener varias interpretaciones y parece razonable exigir mayor precisión. Algunas posibilidades son las siguientes. En primer lugar, podría ser que el típico agente competitivo solamente desee evitar ser la persona que recibe el castigo. Si un agente tuviese tal objetivo (y asignando +1 al beneficio por cumplir el objetivo y 0 por no cumplirlo), interpretaría el juego con la siguiente función de pagos:

$$
\pi_{i}\left(s_{i}, s_{-i}\right)=V\left(s_{-i} \geq s_{i}\right)
$$

Si ese fuera el caso, el análisis teórico indica que todas las coincidencias (desde 180-180 hasta 300-300) devendrían equilibrios de Nash. En este caso, no habría una clara predicción, aunque la apuesta segura sería, claramente, elegir la estrategia inferior, 180 .

$6 \quad$ Nuestro objetivo en esta y la próxima sección del manuscrito es resumir los resultados experimentales más destacables sobre el DVA, pero advertimos al lector que aquí no encontrará una descripción exhaustiva de los procedimientos experimentales utilizados, los cuales fueron ya reportados en la bibliografía correspondientemente referenciada. 
En segundo lugar, podría ser que el típico agente competitivo desee evitar el castigo y también ganar el premio, en cuyo caso, lo que realmente estaría jugando es un torneo. Si un agente tuviese tal objetivo (y asignando +1 al beneficio por el hecho de ganar, -1 por perder y 0 por empatar), evaluaría el juego con la siguiente función de pagos:

$$
\pi_{i}\left(s_{i}, s_{-i}\right)=V\left(s_{-i}>s_{i}\right)-V\left(s_{i}>s_{-i}\right),
$$

lo cual arroja (como en el DV original) un único equilibrio de Nash en (180,180). Aquí la predicción teórica es muy precisa, a saber, tal jugador elegirá 180. Incluso si uno eliminara el supuesto de racionalidad como conocimiento común -y así el agente no pudiera predecir qué elegirá el otro-, nuevamente 180 parecería ser la apuesta segura.

En tercer lugar, podría ser que el típico agente competitivo, además de evitar el castigo y ganar el premio, tuviese como objetivo ganar más dinero que la otra persona. En ese caso su función de pagos sería

$$
\pi_{i}\left(s_{i}, s_{-i}\right)=V\left(s_{-i}>s_{i}>s_{-i}-10\right)-V\left(s_{i}>s_{-i}>s_{i}-10\right)
$$

La predicción teórica nuevamente arroja un único equilibrio de Nash en 180-180. Sin embargo, cuando uno elimina aquí el supuesto de racionalidad como conocimiento común, ya la apuesta segura de 180 deja de tener valor y la elección dependerá crucialmente de la creencia sobre el número que va a elegir el otro agente. Básicamente, lo que este tipo de agente querría es estimar el valor del otro agente y elegir una o unas pocas unidades menos (hasta 9 con los actuales parámetros), con lo cual se quedaría con el premio y con más dinero que la otra persona.

Ahora bien, todas estas posibilidades están abiertas dentro de la hipótesis del objetivo competitivo y probablemente haya personas de estos tipos competitivos mencionados y otros similares (aunque veremos en el resumen del siguiente experimento que el grupo más numeroso parece pertenecer al tercer tipo). Sin embargo, sea cual sea el típico agente competitivo, en todos los casos, se predice que el agente siempre intentará elegir un número inferior al del otro agente, aumentando así la probabilidad de elegir números fuera del rango de estrategias dominantes 290-300.

En un estudio posterior, encontramos evidencia adicional en favor de la hipótesis del objetivo competitivo. Este experimento fue corrido en dos sesiones con un total de 117 participantes, los cuales eran estudiantes de grado de las carreras de Contador Público (sesión 1) y Administración de Empresas (sesión 2) de la Universidad Nacional del Sur, Bahía Blanca, Argentina. En cada una de las sesiones se seleccionaba un cuestionario al azar y se lo enfrentaba a otro cuestionario seleccionado al azar de otro grupo de otra carrera ${ }^{7}$. Anunciamos que a la persona seleccionada del grupo se le pagaría de acuerdo a las reglas del

$7 \quad$ Usamos este procedimiento para preservar el supuesto de que no hay contacto entre los jugadores. Si hubiéramos seleccionado dos personas del mismo grupo, factores sociales podrían haber influenciado el resultado. 
juego, en efectivo y al instante ${ }^{8}$ (véase Freidin, Moro y Tohmé, 2010, y Auday y Moro, 2010). La mitad de los participantes tomaba su decisión después de leer la versión del DVA mencionada anteriormente -de enmarque neutro-, mientras que la otra mitad tomaba su decisión después de leer una versión del DVA de enmarque competitivo descrita a continuación ${ }^{9}$ :

Usted representa a una compañía que compite con otra compañía rival (representada por una persona perteneciente a otra carrera de esta universidad y elegida al azar):

- Usted tiene que elegir el precio de uno de sus productos o servicios en el rango de $\$ 180$ y \$ 300 (los extremos pueden elegirse también).

- La otra compañía, representada por el otro participante, hace lo mismo (leyendo estas mismas instrucciones), pero no se permite ningún tipo de comunicación entre las dos compañías.

- Si usted elige un precio más alto que el de la otra compañía, entonces debe pagar un impuesto de $\$ 5$, es decir, ganará la cantidad que propuso menos $\$ 5$ (la otra compañía recibirá la cantidad que propuso más un bonus de \$5).

- Si usted elige un precio más bajo que el de la otra compañía, entonces recibirá un bonus de $\$ 5$, es decir, ganará la cantidad que propuso más $\$ 5$ (la otra compañía recibirá la cantidad que propuso menos el impuesto de \$ 5).

- Si ambas compañías eligen el mismo precio, cada una recibirá exactamente esa cantidad.

- El precio que elijo es

Nótese que en términos abstractos las reglas son las mismas en ambas versiones del juego. Sin embargo, el resultado fue que, si bien no hubo un efecto global significativo, se encontró un efecto del enmarque en el sentido anticipado en el grupo de participantes que dieron respuestas dominadas. Dentro de este grupo, los participantes de la condición competitiva eligieron valores significativamente más bajos que los participantes de la condición neutral (ver Figura 1). En el grupo de respuestas dominantes, por otra parte, no se registró una diferencia en los promedios de respuestas de las dos condiciones. Así, los resultados pueden interpretarse apelando, por un lado, a la heterogeneidad de motivaciones subyacentes en el DVA y, por otro, al efecto del enmarque competitivo en función de la motivación preponderante en cada participante. En este sentido, vimos que los participantes aparentemente enfocados en la meta de maximizar el dinero (i.e., aquellos que eligieron estrategias dominantes) fueron inmunes al enmarque competitivo. No obstante, la exacerbación de objetivos competitivos a partir del enmarque hizo que aquellos participantes no enfocados en el objetivo

8 Téngase en cuenta que si bien una sola persona recibía el dinero, la cantidad ofrecida era muy sustancial para un estudiante de grado por unos pocos minutos de su tiempo. Para dar al lector una idea, el premio máximo posible era de \$304 y, al momento del experimento, un combo de almuerzo en el buffet universitario rondaba los \$15. De hecho, al anunciar y mostrar el dinero en el inicio del experimento se producía una reacción de ostensible alegría en los participantes.

9 La versión competitiva del DVA es adaptada de una versión del dilema del viajero original presentada en Brañas-Garza et al. (2011). 
de maximizar pagos se movieran en el sentido predicho, es decir, hacia valores significativamente inferiores ${ }^{10}$.

FIGURA 1

PROMEDIO DE RESPUESTAS AL DVA DE AQUELLOS PARTICIPANTES QUE DIERON

RESPUESTAS ESTRICTAMENTE DOMINADAS $(<290)$

COMO FUNCIÓN DEL TIPO DE ENMARQUE.

Barras de error denotan \pm 1 s.e.m.; $* \mathrm{p}<0,05$.

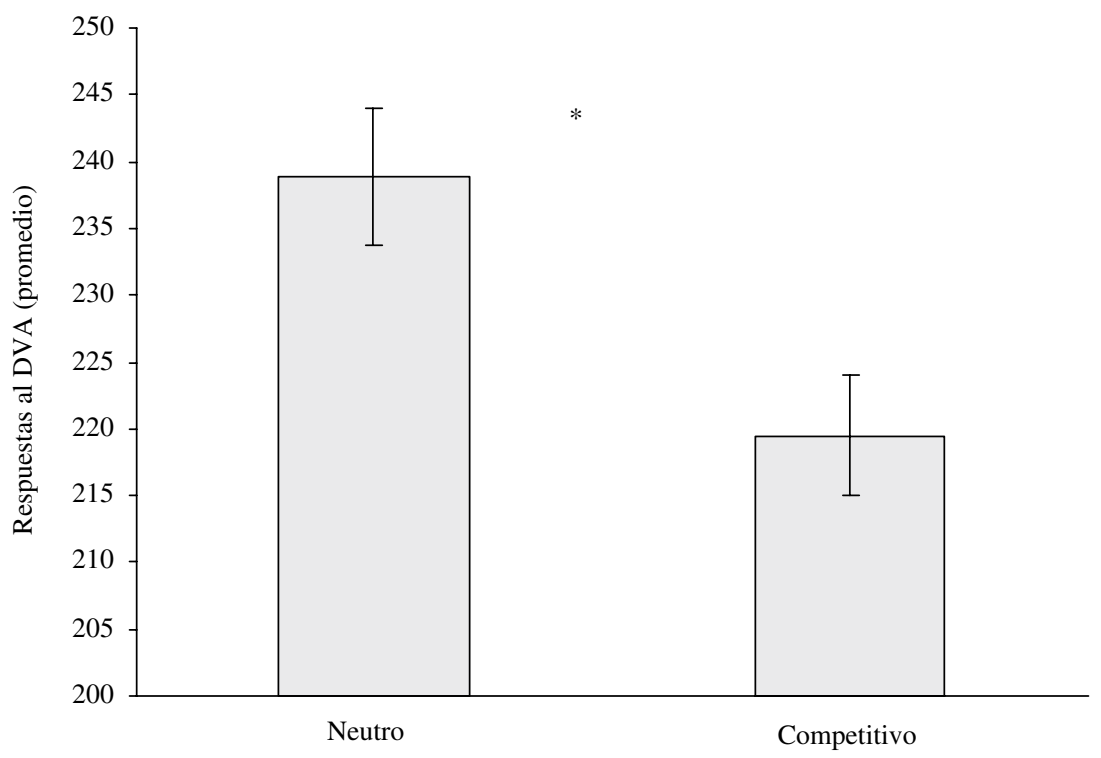

10 Aquí el lector podría preguntarse qué tipo de efecto contextual sería el reportado en este experimento. Para responder esta pregunta, creemos que es muy iluminadora la distinción de Cooper y Kagel (2003). Estos autores distinguen entre un efecto de contexto débil, donde el contexto con significado simplemente actúa como sustituto de la experiencia, acelerando el proceso de aprendizaje y, por ende, la convergencia hacia cierto equilibrio y un efecto de contexto fuerte donde el contexto con significado afecta la selección de equilibrio de los participantes. Si bien nuestros resultados no nos permiten descartar que se trate de un efecto débil (ya que deberíamos haber corrido varias rondas del DVA en lugar de una sola), creemos que el efecto hallado se trataría de un efecto de contexto fuerte. Esto implica que si una persona que eligió un número bajo en la condición con significado probablemente habría elegido un número significativamente más alto en la condición neutra. La justificación de esta propuesta se verá más claramente en la sección 5 donde desarrollemos una explicación integral del fenómeno apelando al concepto de accesibilidad a los objetivos. 


\section{Resumen del Experimento 2: Aspecto Cognitivo}

Ahora bien, la pregunta acerca del rol de las limitaciones cognitivas parece quedar aún abierta como posibilidad explicativa de los resultados descritos en la Figura 1. Más específicamente, los participantes podrían haber elegido estrategias dominadas en el DVA por al menos dos razones distintas. Por un lado, los participantes podrían haber preferido satisfacer algún objetivo alternativo (ej., ganarle al otro) antes que maximizar sus pagos. En tal caso, sus elecciones aún podrían conceptualizarse como la maximización de una determinada función de utilidad asociada a metas distintas del beneficio monetario (hipótesis de preferencia alternativa). Por otra parte, podría tratarse de que las respuestas dominadas fueron efectivamente irracionales en el sentido de que los participantes tal vez valorasen más maximizar sus ganancias que ganarle al otro. Entonces, ¿por qué habrían de buscar de ganarle al otro sacrificando dinero? Podría suceder que los participantes buscaron satisfacer alguna meta competitiva pero sin darse cuenta que, al perseguir tal objetivo competitivo, se estaban perdiendo de maximizar sus pagos (hipótesis de negligencia).

Diseñamos un nuevo experimento $(\mathrm{N}=331)$ para poner a prueba estas dos hipótesis a partir de utilizar tratamientos experimentales en el DVA donde se explicitaba o no la potencial incompatibilidad de las metas en juego, es decir, la meta de maximización de pagos y la meta competitiva (véase Freidin, Moro y Tohmé, 2010). El esquema del diseño general del experimento puede verse en la Figura 2. La clave del diseño fue la siguiente: antes de responder al dilema, en la condición tratamiento se marcó claramente la distinción y potencial incompatibilidad entre dos objetivos, a saber, maximizar pagos y ganar el premio. Por otro lado, en la condición control no se marcó la potencial incompatibilidad entre ambos objetivos, sino que ambos objetivos permanecieron asociados (véase Figura 3). La idea es que si los participantes eligen estrategias estrictamente dominadas $(<290)$ porque prefieren satisfacer un objetivo distinto de la maximización de pagos (hipótesis de la preferencia alternativa), la distinción de metas no debería provocar ningún cambio. Por el contrario, si responden por debajo de 290 justamente porque siguen objetivos competitivos sin darse cuenta de que son potencialmente incompatibles con la meta monetaria (hipótesis de negligencia), el tratamiento debería causar una tendencia hacia la maximización. En otras palabras, de acuerdo a la hipótesis de negligencia, la distinción explícita entre competir y maximizar la ganancia debería dar lugar a un aumento significativo en el promedio de elecciones al DVA al explicitar la potencial incompatibilidad de las metas (bajo el supuesto de que para muchos participantes la utilidad asociada a la meta de maximización de pagos es superior a la de la meta competitiva).

Este experimento tuvo varios ejercicios. El primer ejercicio (denominado "clasificatorio"), presentado inmediatamente después de las reglas del juego, preguntaba a los participantes qué valor elegirían si el otro participante eligiera un valor determinado $X$ (siendo $X$ un valor aleatorio entre 240 y 260). Este ejercicio permitía clasificar a los participantes entre maximizadores iniciales (los que elegían 300) y los no-maximizadores iniciales (los que elegían números menores de 300). Por supuesto, sólo el segundo grupo era de interés y, por tanto, fue el grupo analizado. En el segundo ejercicio, antes de tomar la decisión en el DVA propiamente dicha, a la mitad de los participantes se les daban ejercicios donde 
FIGURA 2

DISEÑO GENERAL DEL EXPERIMENTO SOBRE ASPECTOS COGNITIVOS EN EL DVA

\begin{tabular}{|c|l|l|}
\hline $\begin{array}{c}\text { Número } \\
\text { ejercicio }\end{array}$ & $\begin{array}{l}\text { Condición 1 } \\
\text { Primero distinción }\end{array}$ & $\begin{array}{l}\text { Condición 2 } \\
\text { Primero NO-distinción }\end{array}$ \\
\hline 1 & \multicolumn{2}{|c|}{ Ejercicio de clasificación (si otro jugara...) } \\
\hline 2 & Ejercicios de distinción & Ejercicios de NO distinción \\
\hline 3 & \multicolumn{2}{|c|}{ DVA } \\
\hline 4 & Ejercicio de clasificación (si el otro jugara...) \\
\hline 5 & Ejercicios de NO distinción & Ejercicios de distinción \\
\hline 6 & Ejercicio de clasificación (si el otro jugara...) \\
\hline
\end{tabular}

FIGURA 3

UN EJEMPLO DE EJERCICIOS DE DISTINCIÓN DE OBJETIVOS (PANEL A) Y DE EJERCICIOS DE NO DISTINCIÓN DE OBJETIVOS (PANEL B)

A) Asumiendo que la otra persona va a elegir el número 243,

\begin{tabular}{|l|l|l|}
\hline (Marque con una cruz la opción correcta ) & 240 & 280 \\
\hline ¿Con cuál de las siguientes opciones ganaría el bonus de \$ 5? & & \\
\hline ¿Con cuál de las siguientes opciones ganaría más dinero? & & \\
\hline
\end{tabular}

B) Asumiendo que la otra persona va a elegir el número 236,

\begin{tabular}{|l|l|l|}
\hline (Marque con una cruz la opción correcta $)$ & 235 & 239 \\
\hline ¿Con cuál de las siguientes opciones ganaría el bonus de \$ 5? & & \\
\hline ¿Con cuál de las siguientes opciones ganaría más dinero? & & \\
\hline
\end{tabular}

se distinguía entre los objetivos de maximizar y ganar el premio (véase panel A de la Figura 3) y a la otra mitad, ejercicios similares donde no se distinguía entre dichos objetivos (véase panel B de la Figura 3). La idea era que en la condición de distinción, al ver los objetivos confrontados, los participantes reconocieran de forma explícita que ciertas opciones que permiten ganar el premio no maximizan el beneficio pecuniario. A continuación, los participantes respondían al dilema. Posteriormente respondían nuevamente a un ejercicio clasificatorio (“si el otro jugara X, ¿qué número jugaría usted?") pero cambiando el número concreto con respecto al del ejercicio 1 . Según la hipótesis de negligencia, los 
ejercicios de distinción también deberían aumentar significativamente el promedio de elecciones en dichos ejercicios clasificatorios. A continuación, el grupo que había respondido al ejercicio de distinción respondía el de no-distinción y viceversa, los que habían respondido al ejercicio de no-distinción, respondían uno donde se marcaba la distinción. Finalmente, los participantes respondían a un último ejercicio clasificatorio pero siendo el número dado distinto a los dos anteriores (véase en la Figura 2 el esquema general del diseño). De nuevo, según la hipótesis de negligencia, los ejercicios de distinción, presentados antes o después, tendrían un impacto en el aumento del promedio de elecciones, tanto en el dilema como en los ejercicios clasificatorios.

El procedimiento fue similar al experimento anterior. Nuevamente nuestra muestra fue compuesta por estudiantes de grado de la Universidad Nacional del Sur, pero de una mayor variedad de carreras, a saber, Contador Público, Administración de Empresas, Derecho e Ingeniería Agronómica. El procedimiento fue idéntico al del experimento anterior, excepto que además de sortear una persona por sesión, se sorteaba con un dado el ejercicio a ser pagado, siendo posible cualquiera de los tres ejercicios clasificatorios -en cuyo caso el pago se deducía de inmediato- y el DVA propiamente dicho, en cuyo caso, nuevamente, se seleccionaba el cuestionario de una persona de otra carrera.

¿Cuáles fueron los resultados? Para comenzar -y en concordancia con el experimento anterior- sólo un $45 \%$ de los participantes maximizaron en el primer ejercicio clasificatorio, quedando estos participantes fuera del análisis (ya que el interés estaba en explicar justamente las tendencias del otro grupo). De acuerdo con la hipótesis de negligencia, los ejercicios de distinción tuvieron el efecto esperado, aumentando de manera significativa el promedio de las respuestas tanto en el DVA como en los ejercicios clasificatorios (en comparación con los ejercicios de no-distinción) (véanse Figuras 4 y 5). Adicionalmente, con respecto al ejercicio del DVA propiamente dicho (ejercicio 3 en la Figura 2), el porcentaje de respuestas dominantes fue también significativamente mayor luego de los ejercicios de distinción (68\%) que luego de los ejercicios no-distinción (54\%). Así, el explicitar la distinción entre los dos objetivos principales provocó una tendencia hacia la maximización, dando apoyo a la idea de que muchos participantes eligen opciones dominadas no porque realmente prefieran satisfacer otro objetivo antes que maximizar sus pagos, sino porque parecen no haber considerado en absoluto el objetivo de maximizar, o bien creían erróneamente que dicho objetivo estaba ligado al de ganar el premio. Este resultado brinda apoyo a la tesis de que, además de aspectos motivacionales, hay aspectos cognitivos ejerciendo influencia sobre las tendencias de comportamiento en este contexto.

Sin embargo, esto es sólo una parte de la historia. Si bien el grupo que no maximiza inicialmente es en promedio manipulable en la dirección predicha, no lo es en su totalidad. Una importante porción de participantes $(60 \%$ de los no-maximizadores iniciales, $37 \%$ del total) persistió en no-maximizar aun en el último ejercicio clasificatorio, es decir, cuando ya habían atravesado los ejercicios de distinción entre metas. Bajo el supuesto de que los ejercicios de distinción realmente fueron efectivos en marcar la incompatibilidad de objetivos, se podría argüir que los participantes que persistieron en dar respuestas dominadas realmente prefirieron satisfacer algún objetivo alternativo antes que maximizar el pago. 


\section{FIGURA 4}

PROMEDIO DE RESPUESTAS AL DVA DESPUÉS DE RESOLVER EJERCICIOS

DE DISTINCIÓN (CONDICIÓN D $\rightarrow$ ND) O EJERCICIOS DE NO-DISTINCIÓN (CONDICIÓN ND $\rightarrow$ D).

Barras de error denotan \pm 1 s.e.m.; $* \mathrm{p}<0,05$.

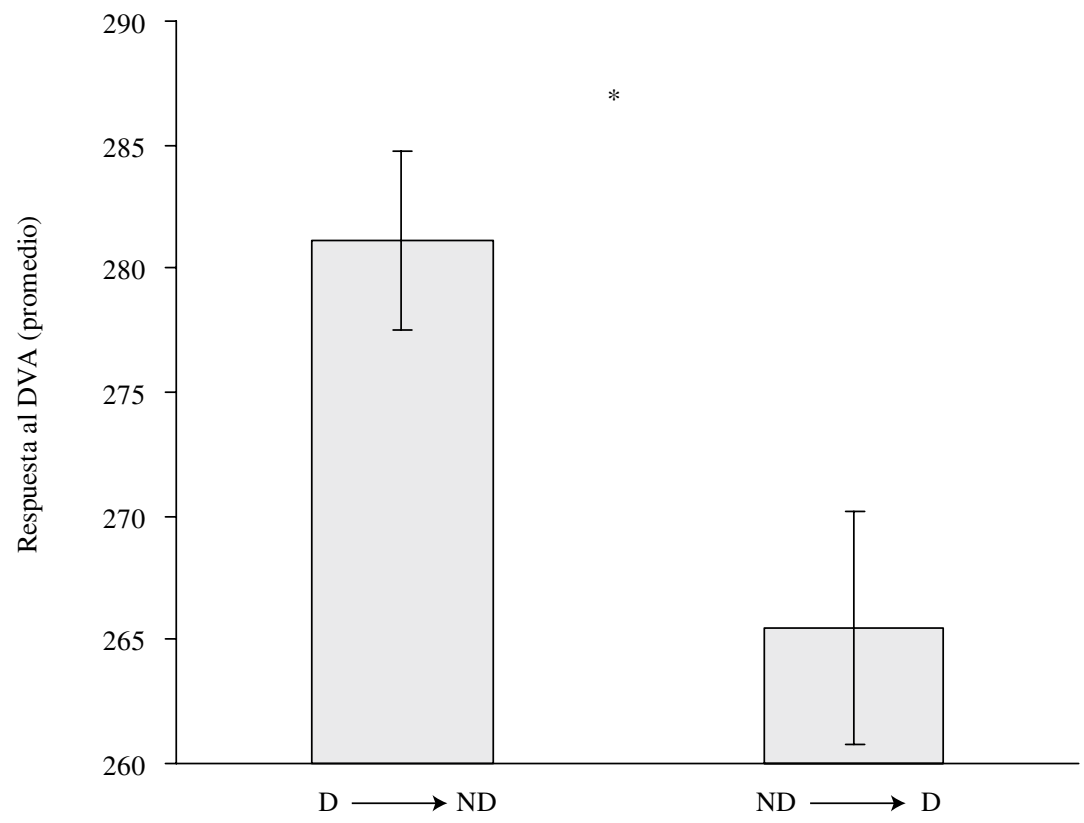

Esto nos lleva a notar nuevamente que -al menos en el contexto del DVA y juegos similares como el dilema del viajero original- hay una gran heterogeneidad de motivaciones subyacentes (siendo la de maximizar pagos sólo una entre ellas). Esto se ve reflejado tanto en las explícitas menciones a objetivos diversos en el estudio preliminar descrito antes (Moro et al., 2010), como en la variedad de respuestas en los ejercicios clasificatorios del estudio de Freidin y colaboradores (2010). En estos ejercicios clasificatorios el participante da su respuesta sabiendo lo que va a elegir el otro jugador, y si bien muchos dan respuestas que maximizan la ganancia monetaria, muchos otros no. Dentro de este grupo de no-maximizadores, un porcentaje relativamente elevado (alrededor del 50\%) elige números de una o muy pocas unidades menores a la respuesta del otro participante, probablemente con motivaciones de índole competitiva. No obstante, si estos participantes estuvieran exclusivamente motivados por ganar el premio y/o evitar el castigo, se esperaría que respondiesen 180 en el ejercicio 3. Definitivamente, las respuestas de los participantes clasificados como "competidores" en el ejercicio 1 (aquellos que eligieron un número menor al dado), no se acomodan a este patrón, ya que dan respuestas en el DVA (ejercicio 3) muy superiores a 180, tanto después de los ejercicios de distinción (promedio \pm 1 e.e.m., $277 \pm 5$ ) como después de los ejercicios de no-distinción (267 \pm 6 ), 
sugiriendo que sus respuestas al DVA podrían resultar de un compromiso entre un objetivo competitivo y ganar tanto dinero como sea posible (en otras palabras, estas personas querrían ganar el premio sin por ello sacrificar en demasía su propio pago). Otra posible interpretación de este resultado es que los agentes, además de evitar el castigo y ganar el premio, hayan intentado también satisfacer el objetivo de ganar más dinero que la otra persona, lo cual los impulsaba a no bajar demasiado sus elecciones. Un grupo importante, aunque menor (alrededor del $35 \%$ ), elige exactamente el mismo número que el otro participante, probablemente con alguna motivación de tipo igualitarista, lo cual se ha registrado en otros juegos como el ultimátum (Dawes et al., 2007). Otra posible interpretación para el comportamiento de este grupo es que sean del grupo competitivo que simplemente quieren evitar el castigo, aunque sin por eso sacrificar en demasía su ganancia monetaria. Finalmente, un grupo más pequeño (alrededor del 15\%) elige números mayores al dado pero sin llegar al máximo 300. En este último grupo de participantes no es claro qué motivación/es puede/n haber subyacido a sus elecciones.

\section{FIGURA 5}

DIFERENCIAS PROMEDIO ENTRE LAS RESPUESTAS A LOS EJERCICIOS CLASIFICATORIOS Y LOS NÚMEROS DADOS (NÚMEROS MÁS ELEVADOS EN LA FIGURA CORRESPONDEN A ELECCIONES MÁS CERCANAS A 300, ES DECIR, A LA MAXIMIZACIÓN DE PAGOS)

Barras de error denotan \pm 1 s.e.m.; $* \mathrm{p}<0,05$.

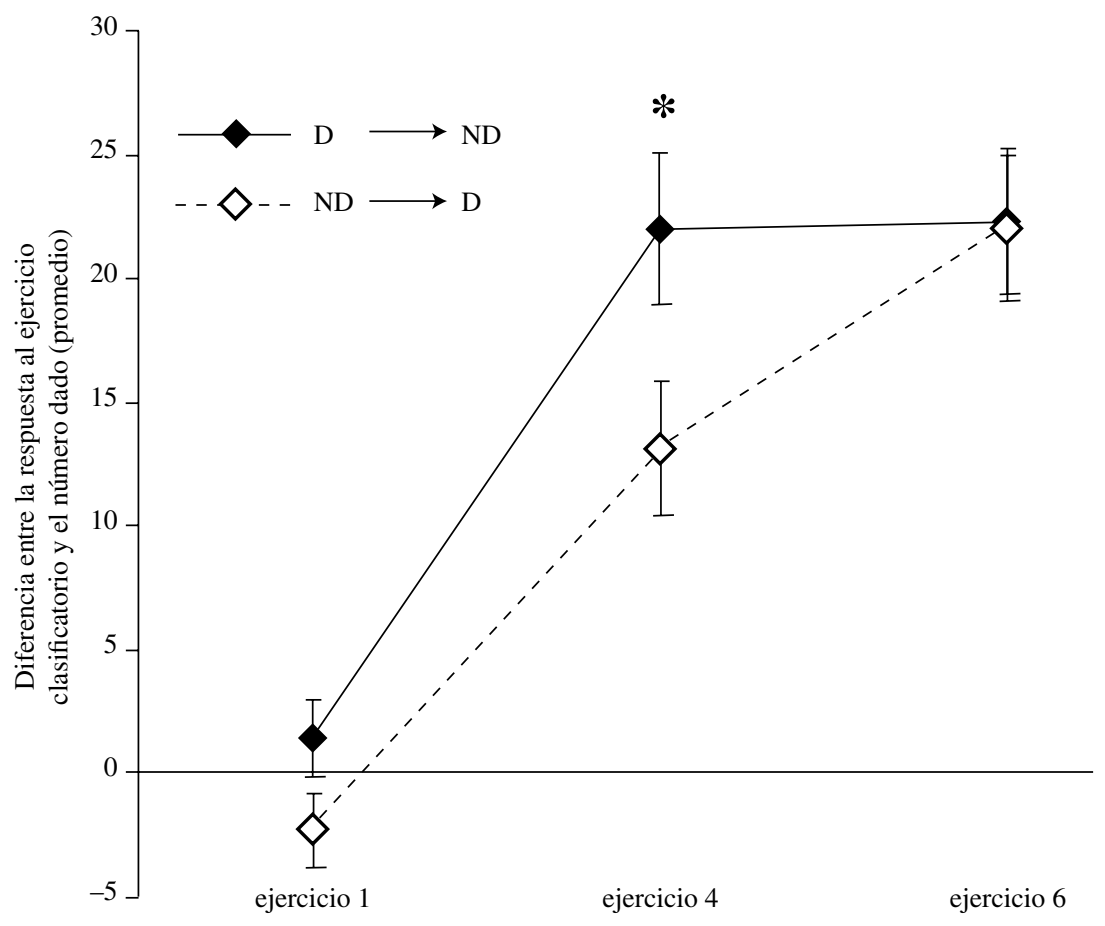


El lector podría preguntarse cuál fue el efecto del tratamiento en el grupo de maximizadores iniciales, quienes habían quedado fuera del análisis. Como era de esperarse, no se encontró ningún efecto significativo, siendo que estos participantes tendieron a seguir maximizando hasta el último de los ejercicios. Por ejemplo, un elevado porcentaje de los participantes clasificados como "maximizadores" en el ejercicio 1 siguieron comportándose como tales en el ejercicio 3 (96\% y $94 \%$ de los participantes de las condiciones $\mathrm{D} \rightarrow \mathrm{ND}$ y ND $\rightarrow \mathrm{D}$ eligieron números $>289$, respectivamente), quedando un $91 \%$ de maximizadores originales hacia el ejercicio 6. Esto concuerda con los resultados del experimento anterior y nos permite resumir los resultados de la siguiente manera. Ante el DVA, hay alrededor de un $40 \%$ de participantes que tienden a maximizar los pagos monetarios y no parecen manipulables a través de factores contextuales (como lo predeciría la teoría económica estándar). El otro grupo es manipulable tanto hacia la maximización (a partir de los ejercicios de distinción) como en contra de la maximización (a partir del enmarque competitivo).

\section{Explicando las Tendencias de Elección en el DVA}

Como se vio en los apartados anteriores, la explicación de las tendencias de elección en el DVA debe involucrar tanto aspectos motivacionales como aspectos cognitivos. La necesidad de incluir aspectos motivacionales puede derivarse de los resultados del experimento 1 (enmarque competitivo vs. enmarque neutro) y por el gran porcentaje de participantes que persistió en no-maximizar en el último ejercicio clasificatorio del experimento 2 (distinción vs. no-distinción de objetivos). El hecho de que la explicación también debe involucrar aspectos cognitivos se ve por el principal resultado del experimento 2 , es decir, que la distinción entre objetivos aumentó la tendencia hacia la maximización. Nótese, por lo tanto, que una explicación cognitivista por sí sola tendría problemas para explicar los resultados del experimento 1 que claramente involucran aspectos motivacionales. Por otra parte, una explicación de tipo motivacional por sí sola se enfrentaría con la dificultad de explicar los resultados del experimento 2. En este sentido, perfectamente uno puede postular funciones de utilidad de tipo competitivo, es decir, que asignen un valor importante a ganar el premio en el DVA, pero en general se espera que dichas preferencias sean estables y, por lo tanto, que no cambien después de resolver ejercicios de distinción de objetivos. ¿Cómo explicar, entonces, las tendencias de elección en el contexto del DVA?

Nuestra propuesta tiene sus raíces en la literatura de la psicología sociocognitiva y tiene la ventaja de combinar las explicaciones cognitivas y motivacionales. Esta literatura presenta evidencia mostrando que las personas parecen no tener preferencias estables y bien definidas como lo supone la teoría económica estándar, sino más bien que las preferencias se construyen en el momento de la elección y son altamente dependientes de factores contextuales (véase Slovic, 1995 y Bettman et al., 1998). Adicionalmente, en muchos contextos, las preferencias están determinadas por los objetivos que se persiguen. El punto crucial es que la accesibilidad en la memoria de un objetivo o meta puede variar en distintos tiempos y contextos (entendiendo accesibilidad como la facilidad con la que un contenido mental viene a la mente, Kahneman, 2003). Los objetivos o metas, como cualquier contenido mental, fluctúan en accesibilidad en distintos 
tiempos y contextos de acuerdo a procesos típicos de activación cognitiva. En otras palabras, la activación de una memoria puede activar/inhibir otra debido a sus conexiones asociativas, lo cual puede hacer a un objetivo o meta más o menos accesible de acuerdo a factores situacionales (Fishbach y Ferguson, 2007). Con estas ideas en mente, consideremos los resultados experimentales en el DVA. Los resultados del experimento 1 pueden interpretarse como que la versión competitiva del DVA aumentó la accesibilidad cognitiva de objetivos competitivos al mismo tiempo disminuyendo la accesibilidad del objetivo de maximizar pagos. Consecuentemente, los participantes que jugaron estrategias dominadas en la condición competitiva eligieron valores significativamente más bajos que sus pares de la versión neutral. Los resultados del experimento 2 pueden interpretarse como que los ejercicios de distinción brindaban mayor accesibilidad al objetivo de maximizar pagos porque mostraban dicho objetivo como distinto del objetivo de ganar el premio. Consecuentemente, los participantes eligieron valores significativamente más altos luego de los ejercicios de distinción que luego de los de no-distinción. En síntesis, los resultados experimentales concuerdan con la idea de que se puede sesgar la accesibilidad al objetivo de maximizar pagos (tanto negativa como positivamente) y los promedios de elección cambian en la dirección predicha. Así, postulando un solo factor se podría dar cuenta de los resultados de ambos experimentos. Más generalmente, nótese que el factor accesibilidad de objetivos que postulamos incluye ambos tipos de aspectos, cognitivos y motivacionales. Hay objetivos y la gente actúa de acuerdo a ellos, por lo que se incluiría un aspecto motivacional. Pero también hay involucrado un aspecto cognitivo: la accesibilidad de contenidos mentales, que puede ser lo suficientemente baja como para que un agente se pierda de satisfacer su objetivo preferido.

Probablemente los investigadores del área propongan explicaciones alternativas a nuestra propuesta y se discutirá acerca de sus méritos relativos. Pero el punto fundamental que queremos remarcar es el siguiente. Sea cual sea la explicación aceptada por la comunidad acerca del comportamiento en el DVA, tal explicación debe poder dar cuenta tanto de aspectos motivacionales como cognitivos.

\section{Conclusión}

Presentamos un nuevo contexto interactivo donde las conductas anómalas de los participantes parecen desafiar los típicos tipos de explicación en el área de teoría de juegos conductual. Propusimos una explicación que da cuenta de las tendencias de elección en cuestión apelando a un concepto proveniente de la psicología sociocognitiva, el concepto de accesibilidad de metas. Pese a lo radical que pueda parecer inicialmente nuestra propuesta, nótese que hay una gran cuota de continuidad con la literatura. En primer lugar, el área de teoría de juegos conductual se ha caracterizado por intentar incorporar factores psicológicos (limitaciones cognitivas, preferencias sociales, etc.) en sus modelos con el fin de incrementar la validez explicativa y predictiva de los mismos (Camerer, 2003, Colman, 2003). En segundo lugar, el hecho de postular un solo factor que explique las tendencias de comportamiento en distintos experimentos apunta a obtener frugalidad en las explicaciones, objetivo por el que se guían gran parte 
de la investigación en el área. Finalmente, el contexto propuesto no parece ser el único que involucraría tanto aspectos cognitivos como motivacionales. Sin ir más lejos, la conducta de los participantes en el dilema del viajero original (DV) parece involucrar ambos tipos de aspectos (ver Basu et al., 2011 y Brañas-Garza et al., 2011). Así, nuestra propuesta no es tan radicalmente distinta como puede parecer a primera vista.

Restaría mencionar que parecería importante intentar incorporar el factor postulado de accesibilidad de metas en modelos formales que permitan explicitar los supuestos detrás del funcionamiento de estos procesos, y así poder poner a prueba su utilidad con mayor rigor. Al mismo tiempo, sería interesante extender el uso de esta noción para explicar y predecir comportamientos aparentemente irracionales en otros fenómenos del área de la economía conductual. Estos desafíos quedan para futuras investigaciones.

Finalmente, si considerásemos el DVA repetido, esto involucraría que a cada par de jugadores, en vez de hacerles jugar el DVA una sola vez, se les haría hacerlo un número de veces, con la expectativa de que sus elecciones cambien en el tiempo como resultado de la experiencia que van adquiriendo al jugarlo. Los análisis teóricos indican que si el número de repeticiones (finito) fuese conocido de antemano por ambos jugadores, ambos elegirían un equilibrio de Nash en cada etapa. En nuestro contexto esto significa que elegirían valores en el intervalo [290, 300]. Si bien en nuestros experimentos esto no ocurre en la única etapa en la que interactúan, cabe esperar que la repetición conduzca en pocas etapas a elecciones dentro de ese intervalo, aun si el número de repeticiones no sea conocido de antemano por los jugadores. Esta hipótesis también podría guiar nuestras investigaciones futuras.

\section{REFERENCIAS}

Auday, M. y R. Moro (2010). "El dilema del viajero: interpretación alternativa y dominación”, presentación en XVI Jornadas de Epistemología de las Ciencias Económicas, Buenos Aires, Argentina, 7-8 de octubre de 2010.

Basu, K. (1994). “The Traveler's Dilemma: Paradoxes of Rationality in Game Theory", American Economic Review, Vol. 84: 391-395.

Basu, K., L. Becchetti \& L. Stanca (2011). "Experiments with the traveler's dilemma: welfare, strategic choice and implicit collusion", Social choice and welfare, in press.

Bettman, J., M. Luce y J. Payne (1998). "Constructive consumer choice processes", The Journal of Consumer Research, Vol. 25: 187-217.

Brañas-Garza, P., M. Espinosa y P. Rey-Biel (2011). “Traveler's types”, Journal of Economic Behavior and Organization, in press.

Camerer, C. (2003). Behavioral Game Theory: Experiments in Strategic Interaction. Princeton University Press.

Camerer, C., T. Ho y J. Chong (2004). "Behavioral Game Theory: Thinking, Learning and Teaching”, en: Huck, S. (Ed.), Advances in Understanding Strategic Behavior: Game Theory, Experiments, and Bounded Rationality: Essays in Honour of Werner Güth. Palgrave MacMillan, 120-180. 
Colman, A. (2003). "Cooperation, psychological game theory, and limitations of rationality in social interaction", Behavioral and Brain Sciences, Vol. 26: 139-198.

Cooper D. y J. Kagel (2003). "The impact of meaningful context in Signalling Games", Journal of Economic Behavior and Organization, Vol. 50; pp. 311-337.

Dawes, C., J. Fowler, T. Johnson, R. McElreath y O. Smirnov (2007). "Egalitarian motives in humans", Nature, Vol. 446: 794-796.

Fehr, E. y U. Fischbacher (2002). "Why Social Preference Matters - The Impact on Non-Selfish Motives on Competition, Cooperation and Incentives", The Economic Journal, Vol. 112: C1-C33.

Fishbach, A. y M. Ferguson (2007). "The Goal Construct in Social Psychology", en Kruglanski, A. y Higgins, E.T. (Eds.), Social Psychology: Handbook of Basic Principles. $2^{\text {nd }}$ Edition. The Guilford Press; 490-515.

Freidin, E., R. Moro, F. Tohmé, F. Contiggiani, M. Miranda, M. Auday y G. Bodanza (2009). "Intuición versus Racionalidad: Explorando los procesos psicológicos en el Dilema del Viajero", presentado en XII Reunión Nacional y I Reunión Internacional de la Asociación Argentina de Ciencias del Comportamiento, Buenos Aires, Argentina, 27-29 de agosto de 2009.

Henrich, J., R. Boyd, S. Bowles, C. Camerer, E. Fehr \& H. Gintis (2004). Foundations of human sociality: ethnography and experiments in fifteen small-scale societies. Oxford University Press.

Kahneman, D. (2003). "A Perspective on Judgment and Choice: Mapping Bounded Rationality", American Psychologist, Vol. 58: 697-720.

Moro, R., G. Bodanza y M. Auday (2010), "El dilema del viajero y sus consecuencias teóricas y Empíricas", en García, P. y Massolo, A. (Eds.). Epistemología e Historia de la Ciencia: Selección de trabajos de las XX Jornadas. Universidad Nacional de Córdoba, Vol. 16: 433-440.

Moro, R., E., Freidin y F. Tohmé (2011). "The Alternative's Traveler Dilemma and the Goal of Payoff Maximization", manuscrito enviado a Journal of Behavioral Decision Making.

Nagel, R. (1995). "Unraveling in Guessing Games: An Experimental Study," American Economic Review, Vol. 85: 1313-1326.

Slovic, P. (1995). "The construction of preference", American Psychologist, Vol. 50: 364-371. 
